\title{
STRUCTURED USE-CASES AS A BASIS FOR SELF-MANAGEMENT OF DISTRIBUTED SYSTEMS
}

\author{
Reza Haydarlou, Michel Oey, Martijn Warnier and Frances M.T. Brazier \\ Faculty of Technology, Policy and Management, Delft University of Technology, Jaffalaan 5, Delft, The Netherlands \\ \{a.r.haydarlou,m.a.oey,m.e.warnier,f.m.brazier\}@tudelft.nl
}

Keywords: Autonomic Computing, Distributed Systems, Self-Management

\begin{abstract}
Automated support for management of complex distributed object-oriented systems is a challenge: selfmanagement of such systems the goal. This paper presents a use-case based approach to self-management of such systems, focusing on autonomic monitoring and diagnosis. The existing notion of use-case has been extended to different levels of system design: explicitly specifying system behavior at different levels, and the relations between these levels, coupling structural models to these descriptions when and where appropriate. The proposed model is illustrated with a small example.
\end{abstract}

\section{INTRODUCTION}

The complexity of software systems, especially distributed systems, increases significantly day by day. As a result, management and maintenance of such complex systems have become a serious problem. Autonomic computing (Ganek and Corbi, 2003; Kephart and Chess, 2003) is a solution proposed to automate management (self-management), and to reduce the cost of maintenance of distributed systems. In the autonomic computing architectural blueprint (IBM Corporation, 2005), a number of architectural building blocks are distinguished of which managed resources and autonomic managers are central.

Figure 1 presents a high-level architecture of a self-management framework (Haydarlou et al., 2006a; Haydarlou et al., 2006b) for distributed objectoriented systems. In line with the autonomic computing architectural blueprint two modules are distinguished: a managed-system and an autonomicmanager. In the approach proposed in this paper the managed-system is an existing distributed object-oriented application extended with sensors and effectors. The autonomic-manager adds the selfmanagement capability to the system. It has two modules: (1) a self-diagnosis module, and (2) a self-adaptation module. The self-diagnosis module continuously checks whether the running application shows any abnormal behavior by monitoring the values it receives from the sensors placed in the application. If so, the self-diagnosis module determines a diagnosis and passes it to the self-adaptation module. The self-adaptation module is responsible for planning actions to resolve abnormal behavior, using the effectors to this purpose.

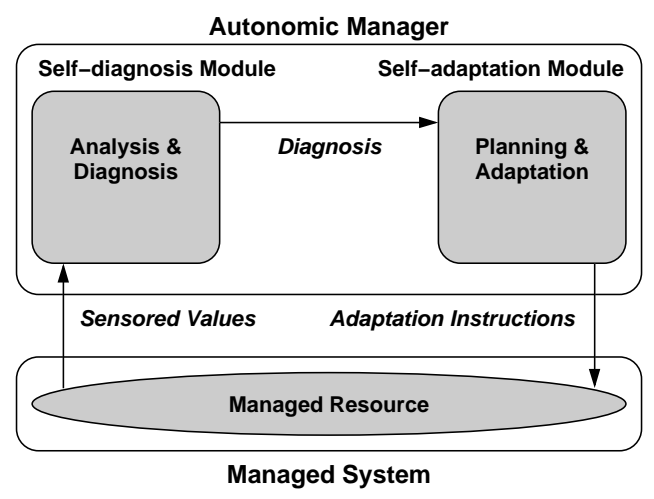

Figure 1: Self-management architecture

This paper presents a self-management framework based on system use-cases, i.e., descriptions of a system's desired behavior. To monitor system behavior and to repair abnormal behavior, the framework uses 
knowledge of the internal structure and dynamic behavior of the running application.

The remainder of the paper is organized as follows: the next section describes the two perspectives (structural and behavioral) that can be used to describe distributed systems. Section 3 argues for the behavioral perspective as the unit of management for distributed systems and Section 4 gives an extension to use-cases that makes them more suitable as a basis for autonomic management. Section 5 gives a brief example of a managed system and illustrates our approach. The paper ends with a discussion and conclusions.

\section{STRUCTURE AND BEHAVIOR}

Conceptually, each system has an internal structure and is designed to exhibit specific behavior. The structure of a system refers to the building blocks of a complex, distributed system, such as servers, runnables, components, and classes. Consider, for example, Trading Systems in a bank environment. These systems consist of several servers, often including at least one web server, (e.g., to allow customers to communicate with the bank), and at least one database server (e.g., to store account information on customers and their shares). The servers together with their (inter)connections form the systems' structure.

System behavior describes the purpose of the (combination of) processes for which a system has been designed. In the above case, the system was designed to process customer transactions to buy and/or sell shares.

\subsection{Structural Perspective}

Many business enterprises run their distributed software systems on a large number of heterogeneous machines each with their own specific operating system(s), middleware, software tools, libraries and support for specific communication protocols. This distributed infrastructure is dynamic, changing continually. Each individual machine has a considerable number of configuration parameters specifying its address, maximum number of allowed connections, connection time-out values, etc.

A typical example of a distributed system can be found in business enterprises that enable customers to reach the company's system through a browser from their homes. To support this functionality, the company runs one or more firewalls to secure their systems. Behind these firewalls run multiple servers, such as the actual web server, an authentication server that ensures that only authorized and authenticated customers can enter, an application server, a database server, message queue server, file server, etc.

Each of these servers can be considered as structural elements within the structure of the entire distributed system. Note that structural elements do not refer to individual hardware components but to software systems. Each of the servers typically run on one or multiple hardware devices (computers) that are all connected through some internal network. For example, a database server could be distributed and/or replicated across multiple computers.

\subsection{Behavioral Perspective}

In practice, the actions a system performs, defining its execution behavior, are designed by multiple parties. Use-cases introduced by Jacobson (Jacobson, 1992), currently a common requirements modeling and structuring instrument, provide a means to describe a system's desired behavior. Although there is no single universally accepted definition of a use-case in the literature, there is a general acceptance that 'a use-case is a collection of possible sequences of interactions between the system and external actors having a set of main goals to be reached with the help of the system' (Cockburn, 2001; Jacobson, 1992; Fowler, 2003).

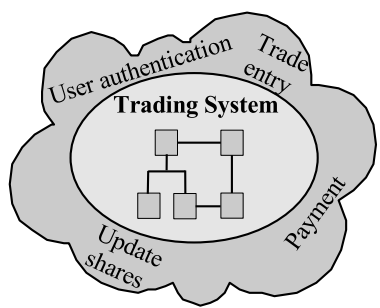

Figure 2: Use-cases of an example Trading System

As an example consider the Trading System, briefly described above. The use-cases defining this system's desired behavior include User Authentication (a user authenticates him/herself to the system), Trade Entry (a user enters a trade in the system), Payment (a user requests the system to administrate his/her payment regarding a trade), and Update Shares (a user requests the system to update his/her shares).

Figure 2 shows a graphical representation of this Trading System with its use-cases. The system is depicted as an ellipse containing a number of squares representing the structural elements of the system. The continuous lines connecting the squares show 
their structural relationships. The example illustrates that system behavior can be considered as a collection of use-cases and a use-case can be seen as a unit of behavior (behavioral element).

Use-cases are most often expressed in a semiformal way (referred to as use-case template or usecase notation). For our purpose, use cases should include the following basic characteristics:

1. A use-case name that uniquely identifies the usecase and clearly expresses its goal.

2. A list of use-case actors. Use-case actors are other systems or persons who initiate interactions with the system to achieve their goals.

3. A use-case trigger which is the event (external, internal, or temporal) that causes a use-case to be initiated.

4. A list of use-case pre-conditions that specifies the conditions (i.e., a certain system state) that must be true for a trigger to meaningfully cause the initiation of a use-case.

5. A list of use-case post-conditions that specifies the desired system states on use-case completion.

6. A list of use-case steps. Use-case steps are either interactions between a system (or part of a system) and an actor, or references to other usecases. References are used to delegate certain subgoals to these referenced use-cases (Kosters et al., 1997).

Note that, for our purpose of system management, a use-case is a description of a process in which a system receives a request, executes use-case steps internally by means of structural elements (e.g., system's sub-systems, components), and produces a response.

\section{UNIT OF SELF-MANAGEMENT}

Self-managing systems often involve multiple autonomic managers. Each autonomic manager focuses on managing one specific part of a system, thereby keeping the autonomic managers themselves manageable. However, one important question is: 'What is the most appropriate unit of self-management?'

The answer to this question depends on how one views a system. The previous section discussed two possible perspectives:

- structural perspective - a system is a collection of structural elements (servers), on each of which a sub-process (an activity) of a business (or nonbusiness) process runs.
- behavioral perspective - a system is a collection of behavioral elements (business or non-business processes), each of which is realized by a number of structural elements.

The choice between these two perspectives leads to the choice of the unit of self-management: associate an autonomic manager with a structural element or a behavioral element (see Fig. 3).

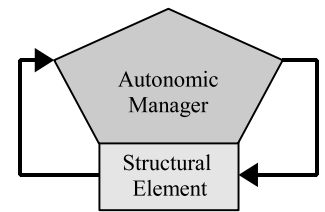

(a)

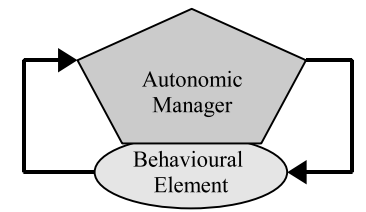

(b)
Figure 3: What is the unit of self-management: (a) a structural element or (b) a behavioral element.

Typically, the structural perspective is chosen, with the structural element as the unit of selfmanagement. For example, each server (e.g., database server, web-server, application server) in a distributed system is managed by a separate autonomic manager.

This paper, however, proposes to choose the behavioral perspective, with the behavioral element (as described by a use-case) as the unit of selfmanagement. Each functional behavior of a system is thus managed by an autonomic manager. The advantage of the behavioral perspective is that autonomic managers can use contextual knowledge to diagnose faulty/abnormal behavior and to recover from system malfunctions (van Harmelen and ten Teije, 1994; Palma and Marín, 2002; Jiang et al., 2009).

\subsection{Availability of Contextual Knowledge}

Diagnosing the root-cause of a system malfunction is a crucial management task (Peischl and Wotawa, 2003; Khanna et al., 2007). Generally speaking, a root-cause is the initiating cause of a causal chain. The primary goal of root-cause diagnosis is to understand why and how a malfunction has occurred, determining which part of a use-case caused a system malfunction, and under which conditions. Without a proper diagnosis, an autonomic manager is not able to construct and perform appropriate remedy plans.

The autonomic manager infers its diagnoses regarding a system malfunction on the basis of information that it obtains from the system during its execution. This information is sent to the autonomic 
manager by sensors instrumented in the system and informs the autonomic manager of the status of specific parts of the system. The obtained information should be analyzed and interpreted in the light of a certain context.

Choosing to associate an autonomic manager with each use-case (i.e., behavioral element, process) provides an autonomic manager with exactly this contextual knowledge whereas associating it with a structural element does not. To clarify, suppose the Trade System has two use-cases: buy shares and sell shares, which buy and sell shares for a customer, respectively. During the execution of either use-case, the system internally uses a number of its servers, including its database server. Both use-cases are examples of behavioral elements and the database server is an example of a structural element.

Associating autonomic managers with structural elements would mean that the database server has a separate autonomic manager that would monitor the activities of the database server. Consequently, if a system malfunction occurs within the database server during the execution of one of the use-cases, the corresponding autonomic manager would detect the failure. However, it would not have information on which use-case was running: it lacks the contextual knowledge. Whereas if the use-case was the unit of selfmanagement, each use-case would have its own associated autonomic manager. In that case, a failure in the database server would be detected by the autonomic manager of the currently executing use-case. In other words, it has the contextual knowledge.

Having contextual knowledge has two benefits. First, after a failure occurs, this knowledge helps to identify the root cause, and consequently, allows remedy plans to recover from the failure and/or to prevent similar failures from occurring in the future. The contextual knowledge provides the diagnosis process with information on which use-case was being executed when the failure occurred, and even the use-case step within the use-case. Knowing how the system was expected to behave (the use-case) is of great help when determining the root cause of a failure.

The second benefit of contextual knowledge is that it also helps to prevent system failures more effectively as it enables an autonomic manager to more accurately monitor the correct and consistent execution of a use-case. To illustrate, selling or buying shares are two different use-cases, with different pre- and post-conditions. For example, the autonomic manager can monitor that the change to the number of shares should be positive if shares are being bought, and negative if shares are being sold ${ }^{1}$. If this check

\footnotetext{
${ }^{1}$ For clarity, a very simple example has been chosen.
}

fails, the autonomic manager can intervene and perform remedy actions before the actual failure occurs in the managed system.

Unfortunately, use-cases in their current form do not relate their use-case steps to the structural elements in which they are executed. Consequently, autonomic managers that base their managed units on use-cases can provide contextual knowledge needed for proper problem determination, but lack the ability to precisely pinpoint the structural element where a malfunction has occurred. In contrast, autonomic managers that base their managed units on structural elements do not have enough contextual information to give a precise diagnosis of faulty systems behavior. The next section discusses how use-cases can be extended to indicate both the activity (in the context of a behavior) that is responsible for a system malfunction, and the place (structural element) where a malfunction has occurred.

\subsection{Extending Use-Cases with Structural Information}

Basing autonomic management on use-cases, which describe the behavior of a system - the perspective chosen in this paper, is not sufficient. Use-cases are executed by processes that run within the managed system. More precise, these processes run within structural elements of the system. Autonomic management requires specification of the relation between processes and the structural elements in a structural model of a system. Note that the executions of different use-cases may be implemented by different processes, which run within different structural elements.

Figure 4 shows that the activation of a specific usecase is mirrored in the activation of related structural elements in the structural model as shown by the highlighted structural elements. Dashed arrows represent the information flow between different structural elements. Continuous arrows represent the input and output of a use-case, respectively. Figure 4(a) depicts the structural elements activated when the User Authentication use-case within the Trading System is executed, and Figure 4(b) shows that other structural elements are activated when the Payment use-case is executed.

Use-case descriptions, as presented in Section 2.2, do not contain references to structural elements. This paper proposes to extend use-case descriptions with the following:

Unfortunately, it does not illustrate the full potential of monitoring the use-case execution with autonomic managers. In practice, such a simple check would typically be done by the managed system itself. 


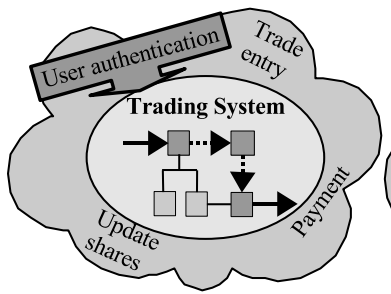

(a)

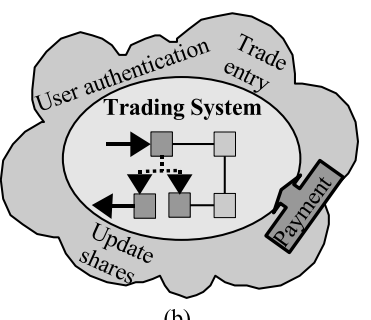

(b)
Figure 4: Use-cases and structural elements

7. A list of use-case structural elements specifying the structural elements responsible for execution of a number of use-case steps. Each use-case is related to a number of structural elements, each use-case step is executed by exactly one structural element.

This extension, in principle, integrates the structural and behavioral perspectives in one use-case description.

For self-management to work, autonomic managers need to be able to monitor a managed system while it is running. Sensors in a managed system provide an autonomic manager with run-time information when executing use-cases. An autonomic manager analyzes this information to determine whether a system is functioning as intended.

Ideally, sensors are placed automatically in the code of the system (Luk et al., 2005) given the usecase descriptions, providing an autonomic manager information on both the behavioral and structural elements involved.

\section{STRUCTURING USE-CASES}

As the number of use-cases of a complex distributed system can be significant, and the complexity of these use-cases can vary noticeably, additional structure is needed. There are two ways to structure use-cases: use-case levels and use-case references.

\subsection{Use-Case Levels}

During system design use-cases are used for requirements modeling and structuring, distinguishing different levels of process design. The first level of process description, the initial use-case specification, describes the behavior of a system as a whole in a system-level use-case. This use-case specification is the basis for the design of a system at more detailed levels. For the purpose of simplicity in this paper specifications at all levels are referred to as use-cases.
Runnable-level use-cases are the next level of specification, defining interactions between systems. These use-cases describe the internal behavior of the system as interactions between runnables (e.g., processes). At a more detailed level component-level use-cases describe behavior as interactions between software components. At class-level use-cases define sequences of method-invocations.

The four above mentioned levels correspond to the different positions often found within an organisation (i.e., system architects, system administrators, functional analysts, system developers).

\subsection{Use-Case References}

Use-case references are used to define the relations between use-cases. Each use-case step (see Section 2.2) can be a reference to another use-case at the same level or at another level (lower or higher). Such references allow a complex use-case to be decomposed into multiple, less complex, (sub)use-cases, which, in turn, can be further decomposed in even smaller use-cases, etc. Furthermore, references also allow use-cases to be 're-used': multiple use-cases can refer to the same (sub)use-case, if they need the same behavior. Figure 5 illustrates this.

Use-case steps, including references to other usecases, are executed by structural elements. A dashed arrow in Figure 5, originating from a structural element (SE $i$ ) and ending at a behavioral element (BE $i$ ), represents the communication between use-cases at the same or different levels. For example, at the runnable level, the dashed arrow (horizontal reference) from SE2 to BE2 shows that the use-case step of behavioral element (use-case) BE1 that runs on structural element SE2 is an invocation of behavioral element BE2 at the same level.

A dashed arrow from a higher level to a lower level, in Figure 5, represents the downward communication between use-cases at different levels. There is a compositional relationship between a structural element executing a downward reference of a use-case at a higher level and the structural elements executing the use-case steps of the referenced use-case at the lower level. For instance, structural element SE1, at the runnable level, consists of structural elements SE4, SE5, and SE6 at the component level. When the downward reference executes on SE1, the system switches to the execution of use-case BE3 executed by the components SE4, SE5, and SE6.

A dashed arrow from a lower level to a higher level represents the upward communication between use-cases at different levels. For instance, use-case BE6, at the class level, needs functionality of a li- 


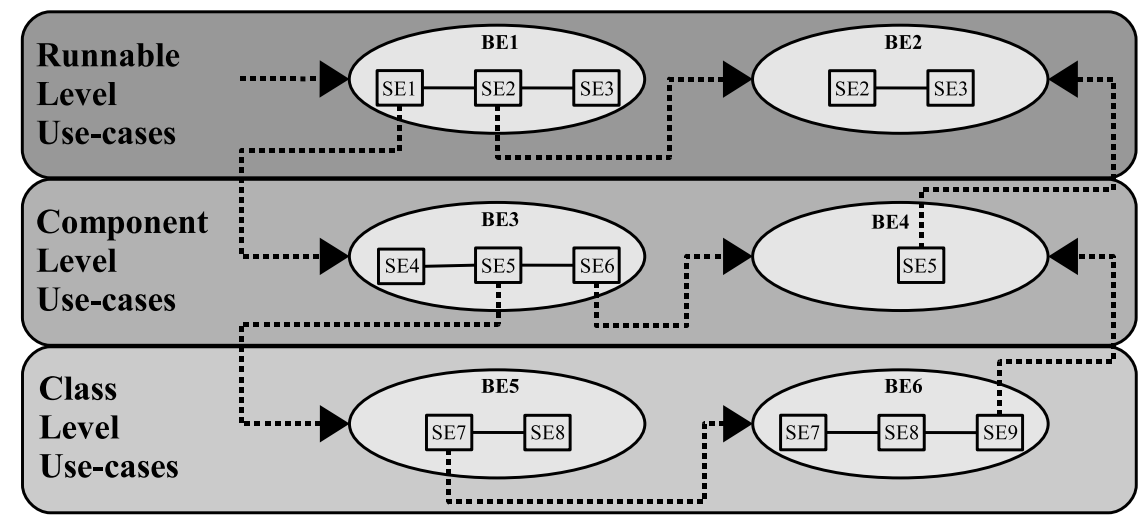

Figure 5: Various use-case levels and their relationships. Horizontal and vertical references between use-cases are shown.

brary (component) that is executed by use-case BE4 in structural element SE5 at the component level. The invocation statement executed within SE9 is an upward reference. Note that there is no compositional relationship between a structural element executing an upward reference of a use-case at a lower level and the structural elements executing the use-case steps of the referenced use-case at the higher level.

Section 5 gives examples of both use-case levels and use-case references.

\subsection{Relationships between Autonomic Managers}

Each autonomic manager manages its own use-case (its managed system). However, as use-cases can reference other use-cases, autonomic managers need to be able to communicate with each other. Success or failure of use-case execution must be communicated to the autonomic manager of the referencing use-case, to locate the root cause of the failure (which may have occurred much earlier than where the system failure surfaced).

Note that each autonomic manager has its own view of a system. Within hierarchical structures, an autonomic manager at a higher level has a different view of the system than lower level autonomic managers and can therefore determine a more general diagnosis.

For example, suppose the autonomic managers are trying to diagnose the root cause of a system malfunction after access to a web page on a web server has failed. A lower level autonomic manager would only detect a connection failed attempt, whereas an autonomic manager at the runnable-level could detect that the internal firewall is (incorrectly) blocking the access.

\section{AN EXAMPLE SCENARIO}

This section presents a simplified version of secure business client authentication for a complex portal system to illustrate multi-level use-case references. Portal systems typically integrate a number of legacy systems, presenting them on the web as a single system. Consequently, the authentication logic for the system as a whole is usually spread out over different sub-systems of the portal system including these legacy sub-systems. As a result, pinpointing a point of failure is often a challenge.

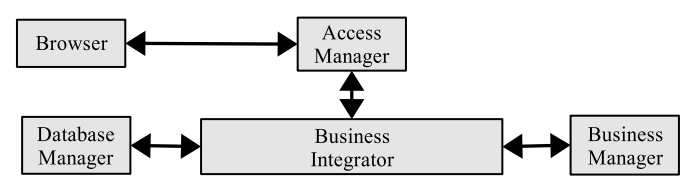

Figure 6: The structural elements of the authentication scenario at runnable level.

Figure 6 illustrates the structural elements of the example scenario and its relations. To access a portal system, business clients provide their certificates to the AccessManager sub-system via the business client's Browser, according to a pre-defined negotiation process (see Figure 6). Upon receiving the client's certificate, the AccessManager verifies the certificate, and passes it to the BusinessIntegrator sub-system. The BusinessIntegrator communicates with the DatabaseManager sub-system, extracts the user's identity (userid), retrieves the password for the given userid, constructs login information (userid/password), and sends it to the BusinessManager sub-system (legacy back-end). The BusinessManager authenticates the user and returns the result of the authentication to the BusinessIntegrator. Finally, the BusinessIntegrator passes the result of 


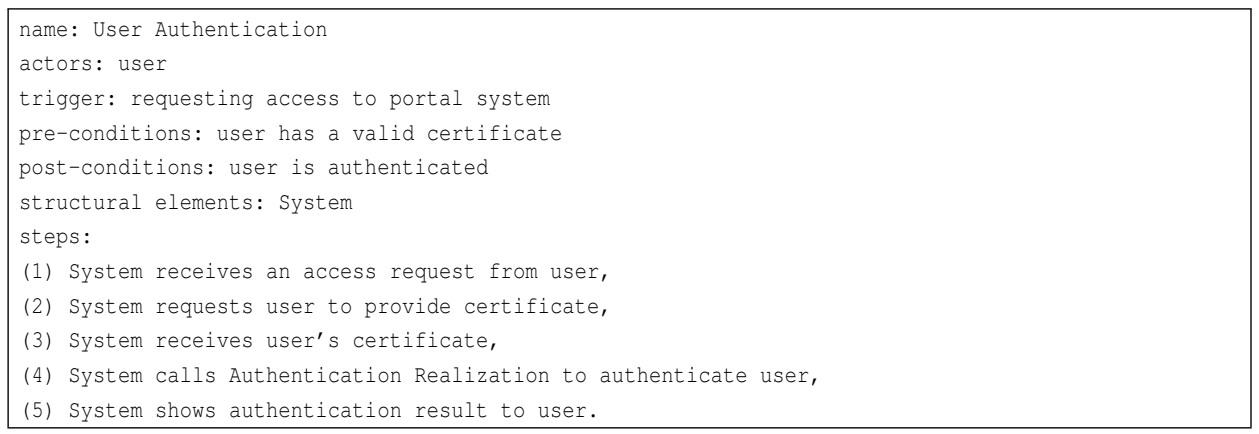

Figure 7: 'User Authentication’ use-case at system level.

the authentication through the AccessManager back to the user's Browser.

Distinguishing a hierarchy of levels in the structure and the behavior of this system on the basis of use-case descriptions, and relating root-causes to these levels can facilitate the diagnostic task by the autonomic managers involved.

Figure 7 shows the authentication process (behavioral element), as a system level use-case. Note that the use-case steps have been intentionally formulated in terms of user and system. A system malfunction may be caused by the fact that a user does not provide a certificate (step 2), the system is not able to authenticate the user (step 4), or the system does not show the authentication result (step 5).

Step 4 in Figure 7 is an example of a use-case reference. This step is an invocation of the Authentication Realization use-case at the runnable level, which is a lower level use-case. In other words, this step is a use-case reference to a use-case at a different level. Figure 8 shows the referenced $A u$ thentication Realization use-case as specified at the runnable level. This use-case is expressed as interactions between runnables. Note that each runnable, as mentioned in Figure 6 (Browser, AccessManager, etc.), may implement one or more use-case steps. The autonomic manager associated with the Authentication Realization use-case communicates with the system-level autonomic manager of the top level 'user-authentication' use-case to determine the rootcause of a system error.

Certain steps in the use-case Authentication Realization are, in turn, references to other use-cases, possibly at other levels. These (sub)use-cases may, in turn, reference other use-cases, etc. In the end, the lowest level use-case will be referenced, specifying the classes and methods involved at the code level.

In summary: the behavior of a complex, distributed system can be described via use-cases that are structured into levels and related through references. By associating autonomic managers with these use-cases, the structure of use-cases allows these autonomic managers to 'zoom in' on the root-cause of a malfunctioning system from high-level use-case descriptions to low-level use-case descriptions.

\section{DISCUSSION AND CONCLUSIONS}

This paper proposes a multi-leveled use-case based approach to self-management. Autonomic managers manage use-cases, and the basic unit of selfmanagement is a single step in a use-case description. The most important argument for this behavioral approach is that autonomic managers can use contextual knowledge to monitor, diagnose and recover from errors. Autonomic managers interact with the structural model of a system - defining relations to structural elements in each and every use-case description.

Structuring use-cases in use-case levels and usecase references helps to streamline self-management even more. Specifying use-cases at different levels requires knowledge acquisition at different levels, by different experts involved in system design and management. Each use-case can be specified by an expert at the appropriate level (i.e., system administrators, functional analysts, system developers, etc.), in (extended) use-case notation.

These notations are suitable for (automatic) transformation into a formal language, for example, in OWL-specifications (Haydarlou et al., 2006a). The OWL-specifications, in turn, can be used to facilitate automatic instrumentation of sensors in the managed system and generation of autonomic managers. A prototype system for transaction management has been implemented in collaboration with the Fortis Bank Netherlands. The first results look promising. 


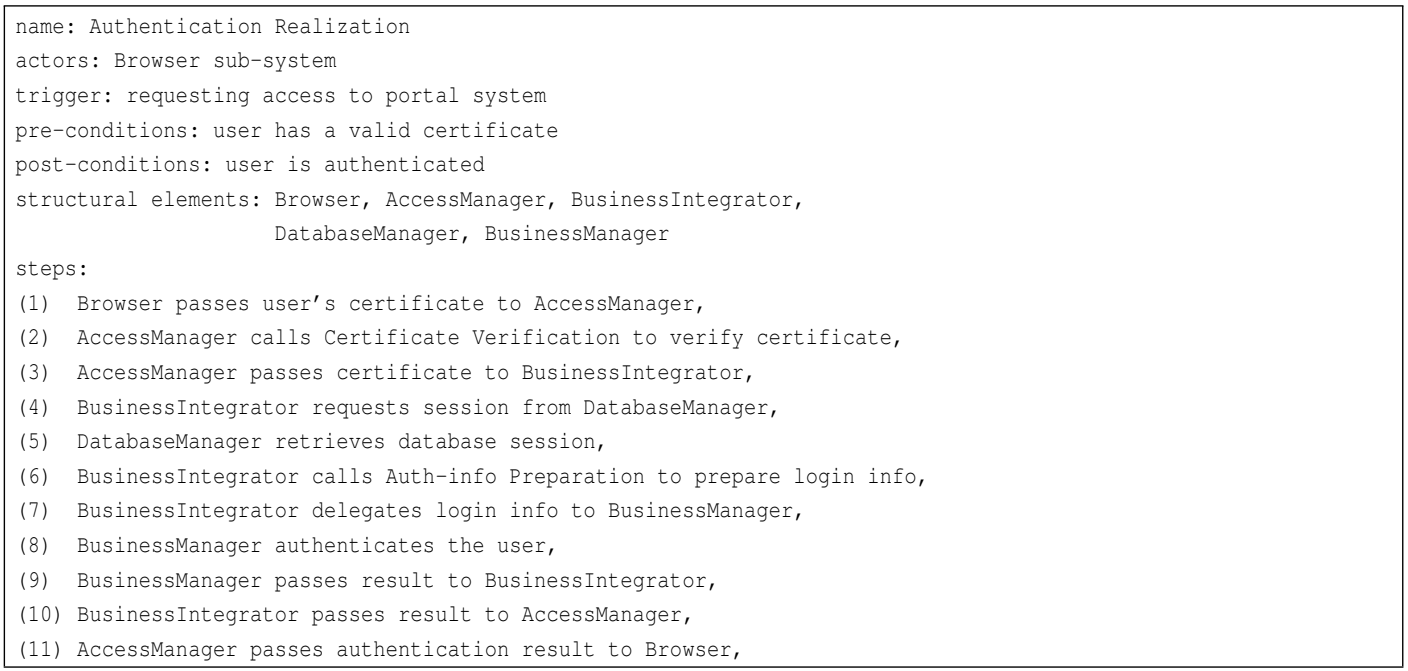

Figure 8: 'Authentication Realization' use-case at runnable level.

\section{REFERENCES}

Cockburn, A. (2001). Writing Effective Use Cases. Addison-Wesley Longman Publishing Co., Inc., Boston, MA, USA.

Fowler, M. (2003). UML Distilled: A Brief Guide to the Standard Object Modeling Language. Addison-Wesley Longman Publishing Co., Inc., Boston, MA, USA.

Ganek, A. G. and Corbi, T. A. (2003). The dawning of the autonomic computing era. IBM Syst. J., 42(1):5-18.

Haydarlou, A. R., Oey, M. A., Overeinder, B. J., and Brazier, F. M. T. (2006a). Using semantic web technology for self-management of distributed object-oriented systems. In Proceedings of the 2006 IEEE/WIC/ACM International Conference on Web Intelligence (WI-06), Hong Kong, China.

Haydarlou, A. R., Overeinder, B. J., Oey, M. A., and Brazier, F. M. T. (2006b). Multi-level model-based self-diagnosis of distributed objectoriented systems. In Proceedings of the 3rd IFIP International Conference on Autonomic and Trusted Computing (ATC-06), Wuhan, China.

IBM Corporation (2005). An architectural blueprint for autonomic computing. Technical report, IBM Corp. White Paper.

Jacobson, I. (1992). Object-Oriented Software Engineering. Addison-Wesley Publishing Company.

Jiang, G., Chen, H., Yoshihira, K., and Saxena, A. (2009). Ranking the Importance of Alerts for
Problem Determination in Large Computer Systems. In Proceedings of the 6th International Conference on Autonomic Computing and Communications (ICAC), pages 3-12. ACM.

Kephart, J. and Chess, D. (2003). The vision of autonomic computing. Computer, 36(1):41-50.

Khanna, G., Cheng, M. Y., Varadharajan, P., Bagchi, S., Correia, M. P., and Veríssimo, P. J. (2007). Automated Rule-Based Diagnosis through a Distributed Monitor System. IEEE Transactions on Dependable and Secure Computing, 4(4):266279.

Kosters, G., Pagel, B.-U., and Winter, M. (1997). Coupling use cases and class models. In Proc. of the BCS-FACS/EROS workshop on "Making Object Oriented Methods More Rigorous", pages pp. 27-30, London, Imperial College.

Luk, C.-K., Cohn, R., Muth, R., Patil, H., Klauser, A., Lowney, G., Wallace, S., Reddi, V. J., and Hazelwood, K. (2005). Pin: building customized program analysis tools with dynamic instrumentation. In PLDI '05: Proceedings of the 2005 ACM SIGPLAN conference on Programming language design and implementation, pages 190-200, New York, NY, USA. ACM Press.

Palma, J. and Marín, R. (2002). Modelling contextual meta-knowledge in temporal model based diagnosis. In ECAI, pages 407-411.

Peischl, B. and Wotawa, F. (2003). Model-based diagnosis or reasoning from first principles. IEEE Intelligent Systems, 18(3):32-37. 
van Harmelen, F. and ten Teije, A. (1994). Using domain knowledge to select solutions in abductive diagnosis. In European Conference on Artificial Intelligence, pages 652-656. 